Artículo / Investigación Article / Research Conteúdo / Investigação

CN: Medico. Esp. Universidad Nacional Experimental de los Llanos Centrales "Rómulo Gallegos". San Juan de Los Morros/Guárico/Venezuela. JM: Universidad Nacional Experimental de los Llanos Centrales "Rómulo Gallegos". San Juan de Los Morros/Guárico/Venezuela. AS: Universidad Nacional Experimental de los Llanos Centrales "Rómulo Gallegos". San Juan de Los Morros/Guárico/Venezuela.

\section{Cirugía convencional y laparoscópica, en colecistectomía}

\author{
Conventional surgical approach vs laparoscopic, in \\ collecistectomy
}

\section{Cirugía convencional e laparoscópica, em colecistectomía}

\author{
Carlos Noval \\ Jomari Montenegro, \\ jomari.mntng@gmail.com \\ Ana María Sanabria, \\ anamariasanabria1408@gmail.com
}

Recibido septiembre 2017 / Revisión octubre 2017 / Aceptado noviembre 2017

\section{RESUMEN}

Introducción. La extracción de la vesícula biliar por procedimiento abierto, contribuye a salvar y mejorar las condiciones de vida, mundialmente. Sin embargo, con la laparotomía se disminuyen las complicaciones, las molestias y la estancia hospitalaria. Objetivo. Determinar los aportes investigativos, de los cirujanos generales, en relación a diferentes técnicas quirúrgicas, en colecistectomía, los factores de riesgo en la técnica convencional/laparoscópica y las técnicas más empleadas por los mismos, en el Hospital Ana Francisca Pérez de León II, en el período de enero 2016 a enero 2017, Miranda/Venezuela. Materiales y métodos. Se realizó una investigación de Campo, de nivel descriptivo, con una muestra de 10 especialistas de Cirugía General, con una revisión literaria, con la recolección y análisis de datos, sustentada en la operacionalización de variables, plasmada en una encuesta y cuya información se compiló a través del programa Excel Microsoft, edición profesional 2007, por distribución de frecuencia y porcentaje, reflejados en cuadros estadísticos y gráficos de barras. Resultados. En un $60 \%$ de los cirujanos afirmaron dar aportes investigativos, usar la laparotomía y abordaje retrogrado, siempre limitados por las distorsiones anatómicas. Conclusión. Los retos quirúrgicos, van de la mano a los avances científicos y sociales, lo que implica realizar las cirugías con la menor agresión al paciente y resultados más rápidos y efectivos.

Palabras clave: Cirugía convencional; laparotomía; colecistectomía

\begin{abstract}
Introduction. The extraction of the gallbladder by open procedure, contributes to save and improve living conditions, worldwide. However, with laparotomy, complications, discomfort and hospital stay are reduced. Objective. Determine the research contributions of general surgeons, in relation to different surgical techniques, in cholecystectomy, the risk factors in the conventional / laparoscopic technique and the techniques most used by them, in the Ana Francisca Pérez de León II Hospital, in the period from January 2016 to January 2017, Miranda / Venezuela. Materials and methods. A field investigation was carried out, at a descriptive level, with a sample of 10 General Surgery specialists, with a literary review, with the collection and analysis of data, based on the operationalization of variables, captured in a survey and whose information was compiled through the Microsoft Excel program, professional edition 2007, by frequency and percentage distribution, reflected in statistical tables and bar graphs. Results. In $60 \%$ of the surgeons they affirmed to give investigative contributions, to use the laparotomy and retrograde approach, always limited by the anatomical distortions. Conclusion. Surgical challenges go hand in hand with scientific and social advances, which means carrying out surgeries with the least possible aggression to the patient and quickest and most effective results.
\end{abstract}

Key words: Conventional surgery; laparotomy; cholecystectomy 


\section{RESUMO}

Introdução A extração da vesícula biliar por procedimento aberto contribui para salvar e melhorar as condições de vida em todo o mundo. No entanto, a laparotomia reduz complicações, desconforto e internação hospitalar. Objetivo Determinar as contribuições dos cirurgiões gerais em relação a diferentes técnicas cirúrgicas, a colecistectomia, factores de risco na técnica convencional / laparoscópica e as técnicas empregues pelo mesmo, no Hospital Ana Francisca Perez de Leon II de investigação, o período de janeiro de 2016 a janeiro de 2017, Miranda / Venezuela. Materiais e métodos. pesquisa de campo, nível descritivo, foi realizado com uma amostra de 10 especialistas em cirurgia geral com uma revisão literária, análise de coleta de dados e de dados, com base na operacionalização de variáveis, refletida em uma pesquisa e cuja informação foi compilada através do programa Microsoft Excel, edição profissional 2007, por distribuição de frequência e porcentagem, refletida em tabelas estatísticas e gráficos de barras. Resultados Em $60 \%$ dos cirurgiões afirmaram dar contribuições investigativas, utilizar a abordagem laparotômica e retrógrada, sempre limitada pelas distorções anatômicas. Conclusão Os desafios cirúrgicos andam de mãos dadas com os avanços científicos e sociais, o que significa realizar cirurgias com a menor agressão possível ao paciente e resultados mais rápidos e eficazes.
CN: Medico. Esp. Universidad Nacional Experimental de los Llanos Centrales "Rómulo Gallegos". San Juan de Los Morros/Guárico/Venezuela. JM: Universidad Nacional Experimental de los Llanos Centrales "Rómulo Gallegos". San Juan de Los Morros/Guárico/Venezuela. AS: Universidad Nacional Experimental de los Llanos Centrales "Rómulo Gallegos". San Juan de Los Morros/Guárico/Venezuela.

Palavras-chave: Cirurgia convencional; laparotomia; colecistectomia

\section{INTRODUCCIÓN}

$\mathrm{D}$ urante décadas, el tratamiento estándar para la litiasis vesicular fue la extracción de la vesícula por medio de procedimiento abierto, lo que contribuyó a salvar muchas vidas y a mejorar las condiciones de numerosos pacientes, por su alta prevalencia mundial (1). Sin embargo, el tratamiento quirúrgico ha evolucionado, disminuyendo la tasa de complicaciones, las molestias posoperatorias y la estancia hospitalaria. De allí, que exista algunas ventajas de la cirugía laparoscópica frente a la cirugía abierta, entre las que resaltan: El menor tamaño de la incisión, con menor complicación, con mejor resultado estético y disminución del dolor. Por lo tanto, la colecistectomía laparoscópica se ha convertido en el método de preferencia, para el tratamiento de la colecistitis, siendo necesario, en muchos de los casos, la conversión a cirugía abierta como recurso alternativo como resolución de la misma, representando esto a nivel mundial con porcentaje que oscilan entre $0.08 \%$ y $18 \%$; pero en Venezuela, esta conversión representa un $2.3 \%$ a $14 \%$.

En 1985, Erich Muhe, en Alemania, realizó la primera colecistectomía por laparoscopia, la cual no fue difundida, pero para 1987, Phillipe Mouret en Francia efectuó otra colecistectomía por vía laparoscópica, la cual fue reconocida mundialmente. Ya para 1986, con la electrónica se desarrolló una mini cámara computarizada de video, con un chip, que se adaptó al endoscopio, de modo que todos en la sala de operaciones pudieran ver el procedimiento y ayudar en forma efectiva. Se inicia entonces el auge de esta técnica de abordaje quirúrgico en el campo de la medicina. En el ámbito latinoamericano fue el venezolano, Dr. Luis Ayala, quien realizó la primera colecistectomía laparoscópica en la década de los 80, en el Hospital de Clínicas Caracas. Por lo tanto, con el transcurso del tiempo, se ha introducido otros elementos, como los trocantes para visualización 
directa de las vísceras y evitar algún daño (2). Actualmente, es un recurso sumamente valioso, tanto en las intervenciones de emergencia como en las programadas.

De allí, que la problemática que hoy se percibe en el área de Cirugía General del Hospital Ana Francisca Pérez de León II, en Caracas/Venezuela, es en cuanto a la aplicación del procedimiento, para realizar la colecistectomía laparoscópica, para el diagnóstico y tratamiento rápido del paciente. Además, el servicio de Cirugía General, cuenta con cirujanos y enfermeras con nivel básico y moderado, de entrenamiento en este procedimiento, aunado déficit en cuanto al material técnico y dotación de los quirófanos. Por lo tanto, el fin de la laparoscopia de emergencia, es el mismo que la cirugía programada. Sin embargo, esta técnica quirúrgica no se escapa de complicaciones al momento de su aplicación. Por lo que, nos basamos en las experiencias, observación y se plasmaron en las encuestas realizadas.

De igual forma, en el Hospital $A B C$ de México, se realizó un estudio que determinó la incidencia de conversión de acuerdo a una población de 1843 pacientes, de los cuales el $2.7 \%$ fueron convertidos (3). También, en el Servicio de Cirugía General del Hospital Universitario Miguel Pérez Carreño, Caracas-Venezuela, se realizó un estudio similar con una muestra de 1970 pacientes intervenidos quirúrgicamente por colecistectomía laparoscópica, en el cual hubo un porcentaje de conversión a cirugía abierta, de $3.2 \%$. Así mismo, en el Hospital Central de Valencia "Dr. Enrique Tejeras", Carabobo-Venezuela, se encontró una incidencia que varío entre 6,45\% a 18,11\% (4).

El finalidad de este estudio fue determinar la distribución por frecuencia sobre los aportes investigativos de los cirujanos generales, en relación a diferentes técnicas quirúrgicas, en cistectomía, sobre factores de riesgo en convencional como en laparoscópica y las técnicas más empleadas por los mismos, en el Hospital Ana Francisca Pérez de León II en el período de enero 2016 a enero 2017.Miranda/Venezuela.

\section{Patologías de la Vesícula Biliar (5)}

- Litiasis: Los cálculos biliares se forman habitualmente en la vesícula, sin embargo, se encuentran cálculos en la vía biliar extra hepática, particularmente colédoco y ampolla de váter. La mayoría de las veces por migración desde la vesícula. Los cálculos de la vesícula constan de un núcleo, constituido principalmente por glicoproteína. Desde el punto de vista del análisis clínico, el componente más importante es el colesterol; también se encuentra bilirrubinato de calcio y carbonato de calcio.

- Cálculos Puros: Pueden ser de Colesterínicos, son únicos, o dos o tres; ovoides, amarillos, de 1 a $2 \mathrm{~cm}$. De diámetro. La vesícula suele no presentar inflamación crónica significativa; frecuentemente presenta colesterolosis. La bilis tiene alto contenido de colesterol. Los Pigmentarios (de bilirrubinato de calcio), son negros o pardos negruzcos, múltiples, fiables, por lo común laminares, de más de diámetro $5 \mathrm{~mm}$. Están asociados con aumento de la bilirrubina no conjugada en la bilis (anemias hemolíticas). Sin lesiones de la vesícula. De carbonato de calcio, muy raros, múltiples, irregulares, gris blanquecino, generalmente sin inflamación de la vesícula.

- Cálculos Mixtos: Son los más frecuentes (80\%), múltiples, pueden alcanzar varias decenas o centenas. Son polifacéticos, verdes parduscos o bien multiformes amarillos. Miden desde fracciones de milímetros a más de $2 \mathrm{~cm}$. 
- Al corte presentan una doble estructura; laminar concéntrica $\mathrm{y}$ cristaloide radiada, con alternancia de estratos blancos amarillentos $y$ parduscos friables. Se asocian siempre con inflamación crónica de la pared de la vesícula.

\section{Consecuencia y Complicaciones de la Litiasis}

- Dolor cólico biliar por obstrucción del cístico, por un cálculo puede provocar una colecistitis aguda. También, la emigración del cálculo por el cístico hasta impactarse en el colédoco o ampolla de Váter, causa ictericia obstructiva y eventualmente colangitis. La litiasis de la vesícula es la más importante en los factores de riesgo de los carcinomas vesiculares.

- Colecistitis Aguda, el 95\% de los casos se asocia con litiasis.

\section{Complicaciones}

- Perforación

- Absceso perivesicular

- Peritonitis biliar o purulenta

- Fistula colecisto-duodenal (más rara colecisto-colónica), por colecistitis aguda con reblandecimiento.

- Colecistitis Crónica: La colecistitis crónica se asocia con litiasis, es una lesión muy común de la vesícula litiásica. Pueden ser una inflamación crónica de comienzo silencioso y de evolución prolongada, o bien ser secundaria a crisis repetidas de colecistitis aguda. Entre las muy diversas formas que puede asumir la vesícula biliar como consecuencia de la colecistitis crónica, destaca 3 variantes peculiares:

\section{Vesícula}

Escleroatrofica: Pequeña, de lumen estrecho y pared fibrosa, amoldada sobre los cálculos del lumen que aparece fuertemente adheridos a ella.
2. Hidrops Vesicular: Vesícula aumentada de volumen, pálida. Al corte, muy distendida por un líquido incoloro de aspecto serosomucoso; un cálculo enclavado en bacinete; pared fibrosa convertida en una delgada lamina.

3. Vesícula en Porcelana: vesícula de pared fibrosa y calcificada; su superficie interna es blanca y lisa.

\section{- Tumores de la Vesícula y de la Vía} Biliar Extrahepática: Las neoplasias más importantes de las vesículas y de los conductos biliares extra hepáticos son: el adenoma y el carcinoma. Los adenomas son generalmente sésiles; de tipo tubular o túbulopapilar, con diferentes grados de displasia. Son raros.

- Carcinomas de la Vesicular Biliar: Es frecuente, ocupa el segundo en mortalidad por cáncer digestivo en chile. Casi siempre se asocia con litiasis y colecistitis crónica. Predomina en mujeres en correspondencia con la mayor frecuencia de litiasis. La mayoría de los carcinomas de la vesícula biliar se origina de nuevo a partir de lesiones precancerosas en mucosa sin adenoma; un bajo porcentaje se origina en adenoma. Los adenomas de la vesícula son muy infrecuentes.

- Carcinoma de Vías Biliares: El carcinoma de la vía biliar extra hepática es menos frecuente que el de la vesícula. Afecta en forma similar a ambos sexos; no tiene relación clara con litiasis; su ubicación, en orden de frecuencia es la siguiente: ampolla de Váter, colédoco y conductos hepáticos; a veces es difícil determinar si se origina en la porción terminal del colédoco, en la ampolla de Váter $o$ en la mucosa duodenal adyacente. Se manifiesta clínicamente por ictericia obstructiva. El carcinoma de la ampolla, tiene un pronóstico 
- menos desfavorable que los demás. Se piensa que la proporción importante de los carcinomas de la ampolla se origina en un adenoma.

\section{Colecistectomía (6)}

Es la extirpación quirúrgica de la vesícula biliar. La operación se realiza para extirpar la vesícula biliar debido a la presencia de cálculos biliares, que causan dolor o infección. Sus síntomas comunes son: Dolor agudo en la parte superior derecha del abdomen que puede pasar a la espalda, al abdomen medio o al hombro derecho, fiebre, náuseas y sensación de hinchazón, ictericia, si hay cálculos biliares bloqueando el conducto biliar.

\section{Tipos}

- Abierta: Se realiza una incisión en el área derecha superior del abdomen, separando las estructuras circundantes, como hígado, vías biliares y las arterias. Carson- De Witt (2008)

- Laparoscopia: Se utiliza instrumentos filosos llamados trocares para hacer cuatro pequeñas aberturas u orificios en el abdomen. Se inserta una aguja especial en el orificio umbilical y se bombea dióxido de carbono. Esto inflara el abdomen para permitir que el contenido se observe más fácilmente. A continuación, luego de diversos procedimientos, se extrae la vesícula biliar a través de uno de los orificios. (Carson-De Witt 2008).

\section{Indicaciones de la Colecistectomía Convencional}

- Dolor abdominal consistente en cólico biliar.

- La presencia de cálculos biliares a través de estudio ecográfico, con una sensibilidad de un 95 a $98 \%$. Los cálculos vesiculares son ocasionalmente identificados en las radiografías en un
$15 \%$ o en las TAC en un $50 \%$, pues los cálculos vesiculares suelen tener suficiente contenido de calcio como para hacer visibles.

\section{Indicaciones de la colecistectomía laparoscópica}

- Los antecedentes del paciente, la evolución de su padecimiento, los resultados de los estudios preoperatorios y los datos de ultrasonografía son algunos elementos con que se cuenta para efectuar la selección del caso.

- Cuando se inicia en la práctica de cirugía laparoscópica, las Colecistectomías deben de ser electivas, sin enfermedades concomitantes, y sin cirugía abdominal previa.

- El tamaño de la vesícula y los cálculos es también un factor que debe tomarse encuentra ya que si existe espacio libre entre los cálculos y la pared, si esta no está engrosada y si son múltiples pero no mayores de 1 centímetro, aumentan las posibilidades de manejar con buenos resultados la vesícula.

La selección de pacientes, debe desaparecer en forma progresiva $\mathrm{y}$ paulatina, para extender la aplicación de la laparoscopia a todos los casos de patología de la vesícula, lo cual se debe complementar y enriquecer con la ejecución selectiva de colangiografía transoperatoria.

\section{Técnicas de la colecistectomía convencional (7)}

Se coloca el paciente en la posición de "Lordosis Forzada" en cuyo medio es posible hacer bascular el hígado y facilitar la exploración del aparato biliar, El estómago e intestino se envuelven en compresas para hacer reintegrados en la cavidad abdominal y se coloca en descubierto la región subhepática, la cual 
está envuelta por adherencia del epiplón gastro-hepático y del colon que cubrirían completamente la vesícula y encierran un solo bloque la región duodeno-pirolico y el aparato biliar a través de esa espesa capa. Se procede posteriormente a la separación metódica de la adherencia con el dedo, ayudado con una compresa, hasta lograr poner en descubierto el fondo de la vesícula, en cuya consecución dirigida hacia el fondo de su lecho, ya cerca del cuello de la vesícula, sus paredes a ese nivel son muy delgadas, e íntimamente pegadas a la adherencia las cuales a desprenderse dejan al descubierto el cálculo. Desde ese momento puede conducir el dedo hacia el hiato de Winslow y explorar las vías principales, ejecutando la maniobra de Kher. Confirmado la colecistitis calculosa, se procede a practicar la colecistectomía. Con el bisturí se hace alrededor de la vesícula una incisión correspondiendo al punto donde se refleja al peritoneo y luego con el extremo de la sonda acanalada al principio; después con la extremidad del dedo, se comienza el desprendimiento favorecido por el plano de clivaje existente entre los dos órganos, luego se aísla la vesícula y el canal cístico. Finalmente, se aísla el canal cístico, con una doble ligadura con catgut. Se coloca un dren de caucho y se cierra el vientre.

\section{Técnicas de la colecistectomía laparoscópica}

\section{Exponiendo el conducto y la arteria} cística: El graspers inmóvil de la posición más latente es utilizado para agarrar la extremidad de la vesícula biliar y para empujarla sobre el borde anterior del hígado por tracción progresiva. En la colecistitis aguda donde la pared vesicular está muy edematosa, se utiliza un instrumento en garra de $5 \mathrm{~mm}$. La bolsa de Hartmann se tira hacia arriba. Esto expone el conducto y la arteria cística así como el conducto biliar común. Es importante esta tracción se mantenga constantemente. En casos difíciles, más prolongados, la manija del graspers se afianza con una abrazadera sobre la piel del abdomen o sobre el campo protector. El paciente entonces se posiciona cabeza abajo.

2. Disecando el conducto y la arteria cística: Una vez que se exponga el campo, la bolsa de Hartmann se agarra con el graspers de trabajo lateral y se tira lateralmente, exponiendo adicionalmente el triángulo de calot el cirujano después pasara un grapers de disección a través de un disector subxyfoideo y comenzara a identificar el conducto cístico. En la colecistitis aguda, las capas edematosas del tejido fino tendrán que ser separadas hacia abajo para exponer el conducto cístico. Con el disector en el portal subxyfoideo se pasa detrás del conducto cístico o bien entre el conducto cístico y la arteria cística. En la mayoría de los casos, el conducto es anterior a la arteria.

3. Seccionando la arteria y el conducto cístico: En esta intersección, se crea la ventana del cístico (es decir, el espacio libre detrás del conducto cístico y la arteria cística). El clip, se inserta vía en trocar subxyfoideo. El conducto y la arteria cística se clipan (3 clip) tan cercanos como sea posible a la vesícula biliar, se inserta la tijera seccionando el conducto cístico y la arteria cística.

4. Disecando la vesícula del lecho hepático: La bolsa de Hartmann ahora esta contraída hacia arriba. Usando el graspers, el lado lateral más bajo de la bolsa de Harmann se debe disecar meticulosamente. El graspers es retirado y sustituido por el gancho de electrocauterio. La vesícula biliar esta 
5. contraída hacia arriba y la tensión se ponen en el plano quirúrgico entre la vesícula y el lecho hepático. La disección se amplía al fondo vesicular. El graspers, que sostiene el muñón del conducto cístico se puede utilizar de vez en cuando para mover por medio de un giro el cuerpo de la vesícula alrededor de graspers inmóvil, que todavía está sosteniendo el fondo vesicular.

6. Extracción de la vesícula biliar: Usando un kit estándar (trocars de 5 a $10 \mathrm{~mm}$ ): un graspers de $10 \mathrm{~mm}$, grande se introduce vía el trocar subxyfoideo. Los dos graspers laterales que sujetan la vesícula, la presentan al graspers grande introducido. La vesícula se saca de la cavidad intra-abdominal a través del mismo sitio trocars. Este sitio trocar se agranda generalmente con pinzas de Pean en algunos milímetros. Usando el kit de los trocars de $5 \mathrm{~mm}$ : la óptica de $5 \mathrm{~mm}$ se retira del trocar umbilical, y se inserta vía el trocar lateral de $5 \mathrm{~mm}$. Un grasper con dientes, entonces se inserta vía entrocar umbilical. La vesícula se agarra y se saca de la cavidad intraabdominal.

Finalmente, la cavidad intra-abdominal entonces se irriga a fondo con Solución salina normal. Todas las piedras que han caído en la cavidad intra-abdominal se recuperan con un morcilador o un fórceps que recupera piedras. Se desinfla en abdomen; los trocars quitados, y los sitios de inserción de los trocars se cierran en la manera habitual.

Técnicas para realizar la

1. Posición Americana: El paciente está en decúbito dorsal y el cirujano se ubica en el lado izquierdo del paciente junto a él, el ayudante que hace de camarógrafo y un asistente al frente, esto es a la derecha del paciente.
2. Técnica cerrada: Se introduce la aguja de Verse a través del ombligo y luego de comprobar que se encuentra en la cavidad, se inicia la insuflación de CO2 en forma progresiva.

3. Técnica abierta: Se hace una disección en la pared abdominal llegando al plano peritoneal, posteriormente se introduce un trócar auto- estático de HASSON y se inicia la insuflación del $\mathrm{CO} 2$ por dicho trócar.

\section{Factores de riesgo de la colecistectomía convencional y laparoscópica}

- Todos los factores de orden anatómico y que por inflamación, infiltración o vecindad, dificultan enormemente la disección de los tejidos. La inflamación y el edema del hilio hepático (porta hepatis), que acompañan a una colecistitis aguda grave y distorsionan la anatomía ductal y vascular.

- El aumento del grosor de la pared por colecistitis crónica o fibrosis.

- La ausencia de mesenterio (vesícula intrahepática).

- La gangrena vesicular que necrosa y licúa los tejidos, lo que impide tomarlos con las pinzas.

- El tamaño del cístico, pues si es muy corto o muy delgado, facilita el riesgo de daño iatrogénico en la vía biliar principal o que se produzca una fistula biliar, y si es muy ancho, lo mejor es no graparlo con clips, sino anudarlo.

- Los cálculos impactados en la pared o en la bolsa de Hartmann.

- Los cambios en la anatomía del conducto y de la arteria cística, que son de diferente orden y manifestación y se presentan hasta el $38 \%$ de los casos.

- Las anomalías o aberraciones en la localización (anormal o atípica), en el tamaño $o$ en el número de las 
- estructuras, que pueden ser muy comunes o muy raras.

\section{MATERIALES Y MÉTODOS}

$\mathrm{S}$

e realizó una investigación de campo, en el lugar y tiempo donde ocurren los fenómenos del objeto de estudio, con un nivel descriptivo, que busca especificar las propiedades importantes de personas, grupos, comunidades o cualquier otro fenómeno que sea sometido a análisis. Miden y evalúan diversos aspectos, dimensiones o componentes del fenómeno a investigar (9). Además, donde el investigador observa la manifestación de algún fenómeno e intenta identificar retrospectivamente sus antecedentes o causas, se manejan datos del pasado (10). Al mismo tiempo, comprende un modelo cuantitativo (11), que pretende describir he interpreta más que medir y predecir; persiguiendo conocer cómo se produce los procesos en su entorno natural y cómo se constituye tales procesos a través de las interpretaciones que hacen de sus actividades los protagonistas (12). Asimismo, para el estudio se usó una muestra de 10 médicos especialistas de cirugía general, para determinar la distribución de frecuencia de los objetivos propuestos, en Hospital Ana Francisca Pérez de León II, ubicado en Petare del estado Miranda, donde actualmente su población se estima en el censo del año 2011 a 2.675.165 habitantes, según XIV censo nacional de población y vivienda 2011 (13).

En relación a la recolección y análisis de datos, se utilizó una encuesta, cuyo instrumento fue un cuestionario de once peguntas cerradas. La encuesta es una técnica fundamental de recolección de datos, para obtener información de una muestra de individuos (14). La información se recolectó en el programa Microsoft Excel edición profesional 2007, por distribución de frecuencia y porcentaje en cuadros estadísticos y gráficos tipo barra. Se procedió a analizar cada variable utilizando la estadística descriptiva $\mathrm{t}$ posteriormente se obtuvieron las conclusiones.

\section{RESULTADOS}

$\mathrm{E}$ análisis e interpretación de los resultados, se basa en técnicas de que relacionan, demonstrar $y$ buscar significados a la información expresada en códigos, verbales e iconos (13).

En aspecto investigativo, como se visualiza (ver tabla 1 y gráfico 1 ), en el ítem 1 (¿Usted ha realizado aportes investigativos en cuanto a métodos quirúrgicos de la colecistectomía?): El 60\% de los especialistas afirmaron haber realizado aportes investigativos, en cuanto a los métodos quirúrgicos, sin embargo, el $40 \%$ negó algún aporte realizado. Con respecto al ítem 2 ( $i$ Usted ha realizado aportes investigativos en cuanto al diagnóstico para la aplicación de la colecistectomía convencional y laparoscópica?): El 60\% negó haber hecho algún aporte, en cambio el $40 \%$ afirmó su aporte investigativo en cuanto al diagnóstico. En el ítem 3 (¿Usted ha realizado aportes investigativos en cuanto al seguimiento del paciente postoperado de una colecistectomía convencional y laparoscópica?): un 50\% "si" han realizado aportes al seguimiento del paciente, a diferencia del otro 50\% "no" lo han realizado. El ítem 4 (¿La colecistectomía convencional es una técnica usada frecuentemente por usted?: El 70\% de los especialistas confirman que "si" es usada con frecuencia por ellos, en cambio el 30\% niega su preferencia. Sin embargo, en el ítem 5 (¿La colecistectomía laparoscópica es una técnica usada frecuentemente por usted?): El 80\% respondió "si" es la técnica más usada por los especialistas, en cuanto que el $20 \%$ niega su preferencia. En cuanto 
a los factores de riesgos, (ver tabla 2 y gráfico 2), en el ítem 6 (¿La distorsión de la anatomía es uno de los factores de riesgo más frecuentes en la colecistectomía convencional y laparoscópica?: El 80\% respondió la opción de "si" es un factor de riesgo frecuente, y un 20\% "no". En cuanto al ítem 7 (¿La litiasis biliar es considerada por usted un factor de riesgo para la colecistectomía convencional y laparoscópica?): $\quad$ El $\quad 70 \%$ de los especialistas afirman ser un factor de riesgo, sin embargo el $30 \%$ lo niega. Finalmente, en cuantos a las técnicas los especialistas del servicio de cirugía general del Hospital Ana Francisca Pérez de León II, dieron su respuesta con respecto al ítem 8 ( $¿$ El abordaje anterógrado en la colecistectomía convencional es usado por usted frecuentemente?): Un $60 \%$ afirmando que el abordaje retrogrado si es usado frecuentemente por ellos, a diferencia del $40 \%$ quienes negaron su preferencia. El ítem 9 (¿El abordaje retrogrado en la colecistectomía convencional es usado por usted frecuentemente?): El 80\% afirma usar este abordaje retrogrado, en cambio el $20 \%$ niega su preferencia. En relación al ítem $\mathbf{1 0}$ ¿ $¿$ Ha realizado estudios en base a las técnicas para el abordaje de la colecistectomía?): el $60 \%$ de los especialistas respondieron a un "si" haber realizado estudios en base a las técnicas para el abordaje de la colecistectomía, y un 40\% niega dichos estudios. El ítem $11 \mathrm{C} \mathrm{Ha}$ realizado algún cambio en las técnicas quirúrgicas de la colecistectomía convencional y laparoscópica?): Un 60\% afirmó cambios en las técnicas quirúrgicas de la colecistectomía convencional y laparoscópicas, mientras que el $40 \%$ lo negó.

Tabla 1. Distribución por frecuencia, sobre los aportes investigativos de los cirujanos generales, en relación a diferentes técnicas quirúrgicas, en cistectomía

\begin{tabular}{ccccccc}
\hline Ítem & & Si & & & No & Total \\
\hline & & $\mathrm{F}$ & $\%$ & $\mathrm{~F}$ & $\%$ & \\
1 & 6 & $60 \%$ & 4 & $40 \%$ & $100 \%$ \\
2 & 4 & $40 \%$ & 6 & $60 \%$ & $100 \%$ \\
3 & 5 & $50 \%$ & 5 & $50 \%$ & $100 \%$ \\
4 & 7 & $70 \%$ & 3 & $30 \%$ & $100 \%$ \\
5 & 8 & $80 \%$ & 2 & $20 \%$ & $100 \%$ \\
\hline
\end{tabular}

Fuente: Montenegro y Sanabria (2017). Datos de la investigación.

Tabla 2. Distribución por frecuencia, sobre factores de riesgo en colecistectomía convencional y laparoscópica, aportados por los cirujanos generales

\begin{tabular}{cccccc}
\hline Ítem & \multicolumn{2}{c}{ SI } & \multicolumn{2}{c}{ NO } & Total \\
\hline 6 & $\mathrm{~F}$ & $\%$ & $\mathrm{~F}$ & $\%$ & \\
7 & 8 & $80 \%$ & 2 & $20 \%$ & $100 \%$ \\
& 7 & $70 \%$ & 3 & $30 \%$ & $100 \%$
\end{tabular}

Fuente: Montenegro y Sanabria (2017). Datos de la investigación. 
Tabla 3. Distribución por frecuencia, sobre las técnicas empleadas por los cirujanos generales

\begin{tabular}{cccccc}
\hline Ítem & \multicolumn{2}{c}{ SI } & \multicolumn{2}{c}{ NO } & Total \\
\hline 8 & $\mathrm{~F}$ & $\%$ & $\mathrm{~F}$ & $\%$ & \\
9 & 6 & $60 \%$ & 4 & $40 \%$ & $100 \%$ \\
10 & 8 & $80 \%$ & 2 & $20 \%$ & $100 \%$ \\
11 & 6 & $60 \%$ & 4 & $40 \%$ & $100 \%$ \\
\hline
\end{tabular}

Fuente: Montenegro y Sanabria (2017). Datos de la investigación.

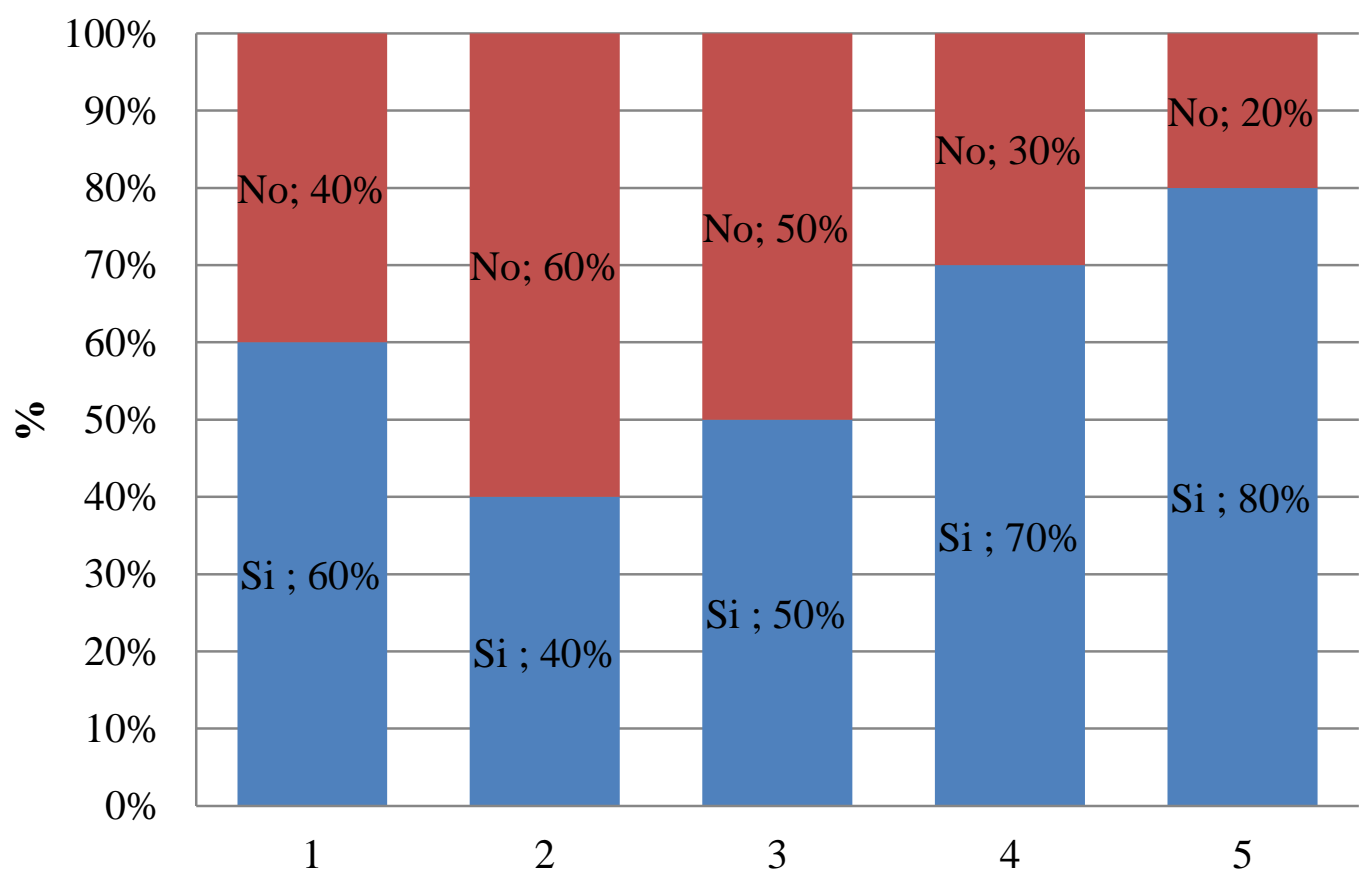

Gráfico 1. Distribución por frecuencia, sobre los aportes investigativos de los cirujanos generales, en relación a diferentes en técnicas quirúrgicas, en cistectomía. (Fuente: Datos de la investigación) 


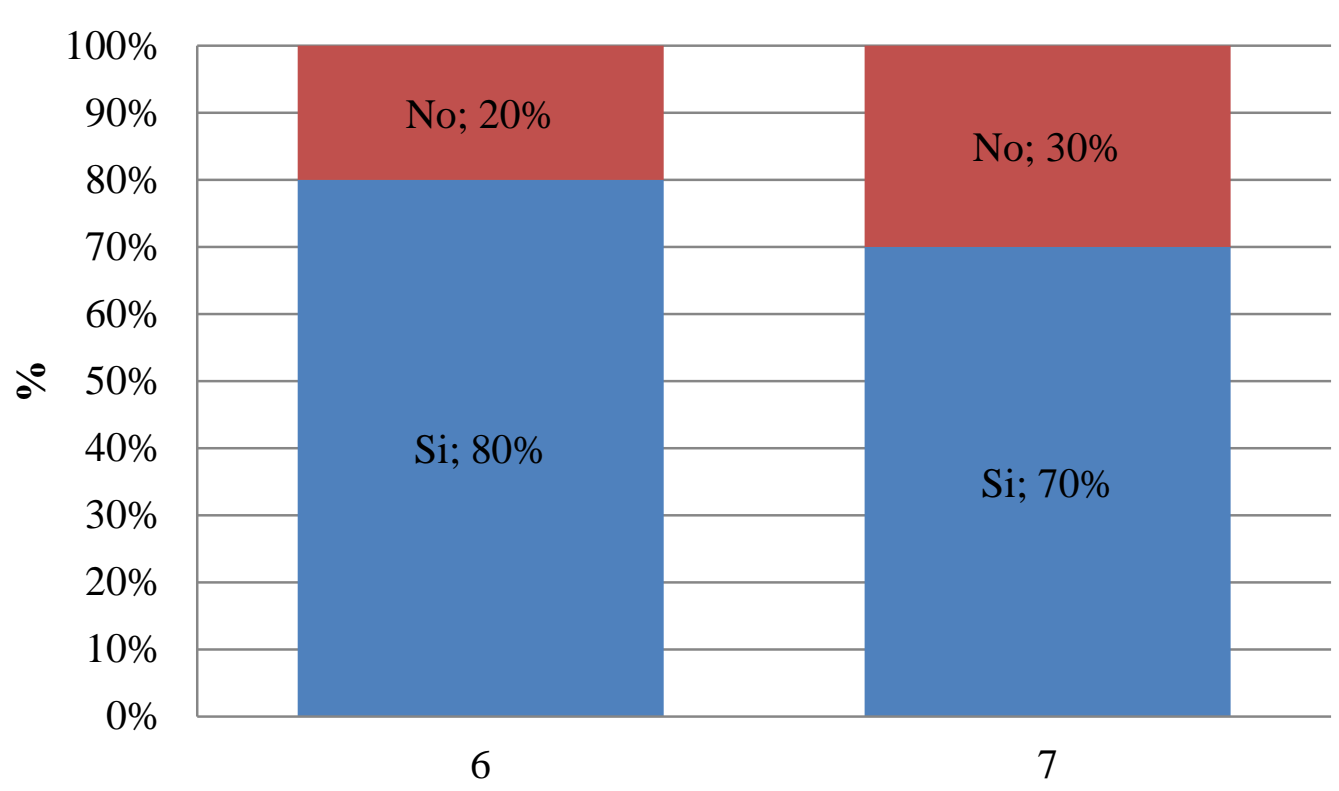

Gráfico 2. Distribución por frecuencia, sobre factores de riesgo en colecistectomía convencional y laparoscópica, aportados por los cirujanos generales

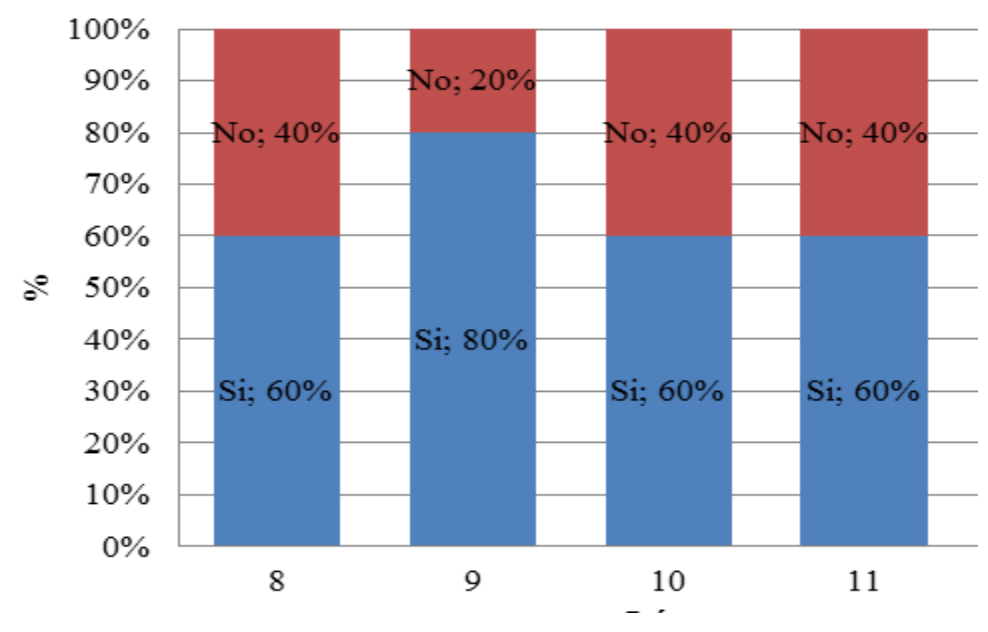

Gráfico 3. Distribución por frecuencia, sobre las técnicas empleadas por los cirujanos generales

\section{CONCLUSIONES}

$\mathrm{P}$ ara finalizar el estudio determinó que los especialistas en cirugía general, aportaron investigaciones al método quirúrgico de la colecistectomía convencional y laparoscópica. También, las variantes anatómicas y el grado de litiasis vesicular, es un factor determinante, para la aplicación de una colecistectomía convencional y una laparoscópica, además los cirujanos, a través de múltiples investigaciones, se han actualizado en nuevas técnicas y abordajes quirúrgicos, frente una colecistectomía convencional y una por laparoscópica, y que los retos quirúrgicos, van de la mano a los avances científicos y sociales, lo que implica realizar las cirugías con la menor agresión al paciente y resultados más rápidos $\mathrm{y}$ efectivos. Por último siempre que existan excelentes precursores, habrá seguidores entrenados, los que constituirán la 
generación de relevo en esta área del sector salud.

\section{REFERENCIAS}

1. Jáuregui, N, Complicaciones de la colecistectomía laparoscópica. Tesis de Grado Inédita. Universidad Occidental "Lisandro Alvarado", Barquisimeto, 2000

2. McAneny D. Colecistectomía abierta. Clínicas Quirúrgicas de Norteamérica. SurgClin N Am 2008; 88:1273-1294

3. Cicero, Alejandra, et al. "Factores que predicen la conversión de la colecistectomía laparoscópica: Cinco años de experiencia en el Centro Médico ABC. Rev Mex Cir Endoscop 2005: 6.26:6-73

4. Lucena Olavarrieta, JR, Dificultades de la colecistectomía laparoscópica en el hombre, Revista de la Facultad de Medicina 2004; 27.2:163168

5. Cervantes CJ, Cirugía Laparoscópica y Toracoscopica. México: McGraw -HillInteramericana, 1997

6. Benítez, Ga, et al. Cirugía Biliar en Venezuela: La Primera Colecistectomía. Parte 1. Revista de la Facultad de Medicina 2003; 26(1):28-30
7. Townsend, Courtney M, Daniel E. Beauchamp, and Kenneth L. Mattox, eds. Sabiston. Tratado de patología quirúrgica. McGraw-Hill Interamericana, SA, 2003

8. American College of Surgeons. Division of Education, Colecistectomía. Extirpación quirúrgica de la vesícula biliar, $\mathrm{s} / \mathrm{f}$

9. Cabrero A., RC. Como hacer un proyecto de investigación. Revista de metodología de la investigación, Universidad de Alicante. 2008; (6): 22-24.

10. Hernández Sampieri R., Fernández Collado C, Baptista Lucio P. Metodología de la Investigación (4ta Edición) 2006

11. Ortiz Uribe, F Diccionario de Metodología de la Investigación Científica (1er Edición). 2004

12. Tamayo M. El proceso de la Investigación Científica: Incluye evaluación y administración de proyectos de investigación (4ta Edición) 2004

13. 1XIV Censo Nacional de Población y Vivienda. Resultados por Entidad Federal y Municipio del Estado Miranda. Diciembre. 2014.

14. Universidad Nacional Experimental Rómulo Gallegos. Área de postgrado. Normas para la Elaboración y Aprobación de Trabajos Técnicos, Trabajos Especiales de Grado, Trabajos de Grado y Tesis Doctorales. San Juan de Los Morros, 2006 